\title{
Structural Parameter Identification in Time Domain using Generalized Hybrid Inversion Method
}

\author{
Xiang-Jian WANG ${ }^{1, a^{*}}$ and Jie CUI ${ }^{2,3, b}$ \\ ${ }^{1}$ Key Laboratory of Earthquake Engineering and Engineering Vibration, Institute of Engineering \\ Mechanics, China Earthquake Administration, Harbin 150080, China \\ ${ }^{2}$ Department of Civil Engineering, Guangzhou University, Guangzhou 510006, China \\ ${ }^{3}$ Earthquake Engineering Research and Test Centre, Guangzhou University, Guangzhou 510405, \\ China \\ a wang.xiangjian@163.com, ${ }^{\mathrm{b}}$ jcui2009@hotmail.com
}

Supported by: the National Science Foundation of China under Grant No. 51208478

Keywords: structural parameter identification; ground motion inversion; extended Kalman filter method (EKF); hybrid inversion method; generalized hybrid inversion method

Abstract: A structural parameter identification method without input information and with limited observations is proposed to identify the stiffness parameters and damping coefficient, to inverse the input information and to estimate the unmeasured responses in time domain. The unique feature of this technique is that it requires only the displacement responses of substructure. This method has two steps: 1) structural physical parameter identification of substructure and input inversion using hybrid inversion method (HIM) with introducing the combination method of the modified LevenbergMarquadt and the singular value decomposition (SVD-mLM) and rectangular window method, and 2) structural physical parameter identification of whole structure and estimation of unmeasured responses using the extended Kalman filter method with weighted global iteration (EKF-WGI) coupled with memory fading filter (MFF) technique and Runge-Kutta (R-K) method. The improved method is called as generalized hybrid inversion method (GHIM) by the authors. The parameter identification and the response estimation are carried out using the proposed method by a numerical example. Three noise contaminated cases of the displacement responses are considered. The robustness, efficiency and accuracy of the new technique are verified by the results.

\section{Introduction}

Dynamic behavior of complicated system often needs to be investigated by system identification (SI) to meet some requirements. SI methods in structural dynamics, in general solve inverse problems to identify properties of a structure from measured data. With the rapid progress in the field of computing technique, use of efficient mathematical tools and experimental technique, SI has become important topic in the field of civil engineering, because SI techniques play an important role in investigating and reducing gaps between the building and their design models. This is also true in structural health monitoring (SHM) for damage detection. And the SI methods are generally used to obtain the dynamic behavior of the structures, to know current status of structural systems against dynamic loads such as earthquake etc.

For dynamic system of civil engineering, the mathematical model is usually known. The SI process is only for the structural parameters, it is called parameter identification. A great amount of researches with various methodologies have been conducted in parameter identification, such as artificial neural network approach (ANN) [1], wavelet analysis method [2], Fourier transform based method [3], finite element-based Iterative Least-squares methods (ILS) [4,5,6], frequency domain decomposition (FDD) [7], natural excitation technique coupled with eigen-system realization algorithm (NExT-ERA) [8], Random decrement technique (RDT) [9], extended Kalman filter technique (EKF) [10,11], and so on.

Since structural physical parameters for damage estimation is intuitive and identified in time domain without cut-off error [5], structural physical parameter identification in time domain become 
a major branch of SI. The writers [5,6] have shown that the finite element-based iterative Least-squares methods with unknown input (ILS-UI) is a good method for structural physical parameter identification in time domain. The method can identify the structural physical parameters and inverse the input time history in time domain simultaneously. J. Li and J. Chen [12] defined the ILS-UI method as hybrid inversion method (HIM).

But HIM needs to observe the dynamic response of every dynamic degree of freedom (DDOF) for identifying all the structural parameters and inversing the input information. In the dynamic testing of the real building, measuring the dynamic responses of all DDOFs is often difficult. And the extended Kalman filter method with weighted global iteration (EKF-WGI) doesn't need to measure the dynamic response of all DDOFs. For solving the problem, the ILS-UI, EKF-WGI and the substructure method are composed together for structural parameter identification and input inversion in time domain with unknown input and limited observations. In this paper, the composed method is called as generalized hybrid inversion method (GHIM) for the same objectives (structural parameter identification and input inversion) of HIM but with limited observations. And the method is developed with introducing some techniques and used to identify the structural physical parameters and inverse the ground motion in time domain.

\section{Generalized Hybrid Inversion Method (GHIM)}

The basic framework of GHIM is: firstly, identify the structural parameters of the sub-structure and inverse the input information with limited observations using HIM; secondly, estimate the structural papers of the whole structure and unknown dynamic responses with the inversed input time history using EKF-WGI. The method will be given as the following [13].

The governing equation of motion of a multiple degree of freedom (MDOF) system can be written in matrix form as

$$
\mathbf{M} \hat{\mathbf{x}}+\mathbf{C} \mathbf{x}+\mathbf{K x}=\mathbf{f}(t)
$$

where $\mathbf{K}, \mathbf{C}$ and $\mathbf{M}=$ stiffness, damping and mass matrix of the structure, respectively; $\mathbf{x}$, $\mathbf{x}$ and $=$ displacement, velocity and acceleration vectors, respectively; $\mathbf{f}(t)=$ excitation force vector.

Hybrid Inversion Method of the Substructure. In general, the mass changing of damaged structure is very small, the mass can be assumed as known. By using the finite element technique, the parametric identification equation can be written as

$$
\mathbf{P}=\mathbf{F}-\mathbf{M} \mathbb{E}=\mathbf{H \theta}
$$

where $\mathbf{P}=\left[\begin{array}{llll}\mathbf{P}_{1} & \mathbf{P}_{2} & \mathrm{~L} & \mathbf{P}_{L}\end{array}\right]^{\mathrm{T}}$ is the excitation force-related vector; $\mathbf{H}=\left[\begin{array}{llll}\mathbf{H}_{1} & \mathbf{H}_{2} & \mathrm{~L} & \mathbf{H}_{L}\end{array}\right]^{\mathrm{T}}$ is the response information matrix; $\boldsymbol{\theta}$ is the parameter vector, including stiffness parameters and damping coefficients; $L$ is the number of sampling points.

The identified values of parameters using Least-squares method (LS) can be written as

$$
\hat{\boldsymbol{\theta}}=\left(\boldsymbol{H}^{T} \boldsymbol{H}\right)^{-1} \boldsymbol{H}^{T} \boldsymbol{P}
$$

For $n$-story shear-type structure under support excitation, at $i$-th node, the left item of the Eq. 2 can be stated as

$$
p_{i}(k)=f_{i}(k)-m_{i} \aleph_{i}(k)=-m_{i} \bigotimes_{g}(k)-m_{i} \aleph_{i}(k) \quad(i=1,2, \mathrm{~L}, n)
$$


where $m_{i}$ is the lumped mass of $i$-th node; $\mathbb{N}_{g}(k)$ is the acceleration of ground motion at the time of $t=k \cdot \Delta t ; \&(k)$ denotes the relative acceleration response; $n$ is the total number of DDOFs. The calculation steps of hybrid inversion method are listed as follows:

(1) Give the initial values of parameters, $\tilde{\boldsymbol{\theta}}_{0}$. Calculate the force-related vector, $\widetilde{\mathbf{P}}$, using Eq. 2.

(2) Using Eq. 4 and statistical average algorithm (SAA), the ground motion can be inversed as

$$
\left.\bar{g}(k)=\frac{1}{n} \sum_{i=1}^{n}(k)+\frac{\tilde{p}_{i}(k)}{m_{i}}\right)
$$

(3) Using Eq. 4, the force-related vector can be modified as $\hat{p}_{i}(k)=-m_{i} \bar{\bigotimes}_{g}(k)-m_{i}(k)$.

(4) Using LS, the parameters are identified as $\tilde{\boldsymbol{\theta}}_{1}=\left(\mathbf{H}^{T} \mathbf{H}\right)^{-1} \mathbf{H}^{\mathrm{T}} \hat{\mathbf{P}}$.

(5) $\tilde{\boldsymbol{\theta}}_{0}=\tilde{\boldsymbol{\theta}}_{1}$, repeat all the steps until meet the stopping criteria.

Under the conditions of limited observations and unknown input, the substructure technique needs to be introduced to identify the structural parameters and inverse the input using HIM. In order to obtain the structural parameters of substructure and input information with minimum dynamic responses, the substructure including the top two DDOFs is considered in this paper. The motion equation of the substructure is

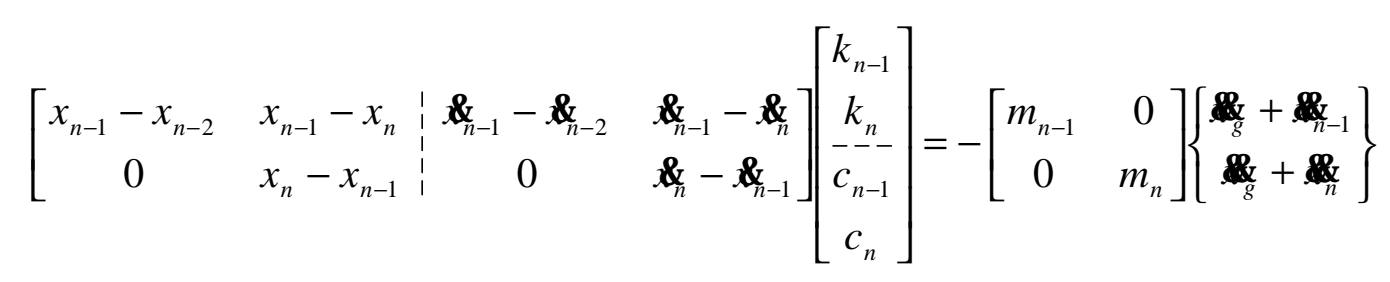

The equation can be expressed as Eq. 2, and the vectors of Eq. 2 for substructure are written as

$$
\begin{aligned}
& \mathbf{H}(k)=\left[\begin{array}{lll}
\mathbf{H}_{k} & \mathbf{H}_{c}
\end{array}\right]=\left[\begin{array}{cc:cc}
x_{n-1}-x_{n-2} & x_{n-1}-x_{n} & \hat{x}_{n-1}-\hat{x}_{n-2} & \hat{\boldsymbol{x}}_{n-1}-\hat{\boldsymbol{x}}_{n} \\
0 & x_{n}-x_{n-1} & 0 & \hat{\boldsymbol{d}}_{n}-\boldsymbol{f}_{n-1}
\end{array}\right] \\
& \boldsymbol{\theta}=\left[\begin{array}{l:l}
\boldsymbol{\theta}_{k} & \boldsymbol{\theta}_{c}
\end{array}\right]^{\mathrm{T}}=\left[\begin{array}{ll:ll}
k_{n-1} & k_{n} & c_{n-1} & c_{n}
\end{array}\right]^{\mathrm{T}} \\
& \mathbf{P}(k)=-\left[\begin{array}{cc}
m_{n-1} & 0 \\
0 & m_{n}
\end{array}\right]\left\{\begin{array}{c}
\frac{1}{g}+1 \\
g
\end{array}\right\}
\end{aligned}
$$

Eq. 6 and Eq. 7 show that the dynamic responses of top three DDOFs should be known. One of three responses (displacement, velocity and acceleration) should be measured, and the other two responses can be reconstructed from the measured one.

For Rayleigh damping or stiffness damping case, the parametric identification equation is nonlinear. The combination method of the modified Levenberg-Marquadt and the singular value decomposition (SVD-mLM) [5] is introduced for solving the nonlinear Least-squares iteration problem. In the first step, the physical parameters of the substructure and the ground motion will be obtained with limited observations and unknown input.

Extended Kalman Filter Method with Weighted Global Iteration of the Whole Structure. For physical parameter identification of dynamic system, the physical parameters are extended into the state vector, and estimated using Kalman filter method, that is called extended Kalman filter (EKF). The EKF technique is explained and as follows [13,14]: 
Through Eq. 1, the state equation of the dynamic system is expressed as

$$
\mathbf{X}=\left\{\begin{array}{l}
\mathbf{X}_{1} \\
\mathbf{x}_{2} \\
\mathbf{x}_{3} \\
\mathbf{X}_{4}
\end{array}\right\}=\left\{\begin{array}{c}
\mathbf{X}_{2} \\
\mathbf{M}^{-1}\left[\mathbf{f}(t)-\mathbf{C}\left(\mathbf{X}_{4}\right) \cdot \mathbf{X}_{2}-\mathbf{K}\left(\mathbf{X}_{3}\right) \cdot \mathbf{X}_{1}\right] \\
\mathbf{0} \\
\mathbf{0}
\end{array}\right\}
$$

where, $\mathbf{X}=\left[\begin{array}{llll}\mathbf{X}_{1} & \mathbf{X}_{2} & \mathbf{X}_{3} & \mathbf{X}_{4}\end{array}\right]^{\mathrm{T}}=$ state vector; $\mathbf{X}_{1}=\left[\begin{array}{lll}x_{1} & \mathrm{~L} & x_{n}\end{array}\right]^{\mathrm{T}}$ and $\mathbf{X}_{2}=\left[\begin{array}{lll}\hat{x}_{1} & \mathrm{~L} & \boldsymbol{x}_{n}\end{array}\right]^{\mathrm{T}}=$ displacement and velocity vectors, respectively; $\mathbf{X}_{3}=\left[\begin{array}{lll}k_{1} & \mathrm{~L} & k_{l}\end{array}\right]^{\mathrm{T}}$ and $\mathbf{X}_{4}=\left[\begin{array}{lll}c_{1} & \mathrm{~L} & c_{l}\end{array}\right]^{\mathrm{T}}=$ stiffness and damping vectors in element-level, respectively. $l=$ total number of elements; $x_{i}$ and $\hat{x}_{i}=$ displacement and velocity at the $i$ th DDOF; $k_{i}$ and $c_{i}=$ stiffness and damping parameters in element-level that need to be identified, and they are assumed not to change with time during the identification process. $\mathbf{K}\left(\mathbf{X}_{3}\right)$ denotes stiffness matrix is the function of the state vector $\mathbf{X}_{3} ; \mathbf{C}\left(\mathbf{X}_{4}\right)$ denotes damping matrix is the function of the state vector $\mathbf{X}_{4}$. In general, Eq. 4 can be written as

$$
\hat{\mathbf{X}}(t)=\mathbf{g}[\mathbf{X}(t), \mathbf{f}(t), t]+\mathbf{L}(t) \cdot \mathbf{w}(t)
$$

Eq. 9 is the nonlinear continuous state equation, where $\mathbf{X}(t)=$ continuous state vector, $\mathbf{g}[\bullet]$ denotes the nonlinear function of $\mathbf{X}(t), \mathbf{L}(t)=$ matrix of system noise, $\mathbf{w}(t)=$ system noise vector.

Suppose the dynamic response of the structure is measured at time $t_{k+1}=(k+1) \Delta t$, where $\Delta t=$ time interval between measurements. Then the observation equation at time $t_{k+1}=(k+1) \Delta t$ can be expressed as

$$
\mathbf{Z}_{k+1}=\mathbf{h}\left[\mathbf{X}_{k+1}, k+1\right]+\mathbf{V}_{k+1}
$$

Eq. 10 is the nonlinear discrete observation equation, where $\mathbf{X}_{k+1}=\mathbf{X}\left(t_{k+1}\right)=$ discrete state vector, $\mathbf{h}[\bullet]$ denotes the nonlinear function of $\mathbf{X}_{k+1}, \mathbf{V}_{k+1}=$ observation noise.

Some assumptions are given as follows:

$$
\begin{aligned}
& E[\mathbf{w}(t)]=0, \quad E\left[\mathbf{V}_{k}\right]=0 \\
& \operatorname{Cov}[\mathbf{w}(t), \mathbf{w}(\tau)]=\mathbf{Q}_{\mathbf{w}}(t), \quad \operatorname{Cov}\left[\mathbf{V}_{k}, \mathbf{V}_{j}\right]=\mathbf{R}_{k} \boldsymbol{\delta}_{k j}, \quad \operatorname{Cov}\left[\mathbf{w}(t), \mathbf{V}_{j}\right]=\mathbf{0} \\
& E\left[\mathbf{X}_{0}\right]=\overline{\mathbf{X}}_{0}, \quad \operatorname{Var}\left[\mathbf{X}_{0}\right]=E\left[\left(\mathbf{X}_{0}-\overline{\mathbf{X}}_{0}\right)\left(\mathbf{X}_{0}-\overline{\mathbf{X}}_{0}\right)^{\mathrm{T}}\right]=\mathbf{P}_{0} \\
& E\left[\mathbf{X}_{0}, \mathbf{w}(t)\right]=0, \quad E\left[\mathbf{X}_{0}, \mathbf{V}_{k}\right]=0
\end{aligned}
$$

where $\mathbf{Q}_{\mathbf{w}}(t)$ and $\mathbf{R}_{k}=$ covariance matrixes of system noise and observation noise, respectively; $\boldsymbol{\delta}_{k j}=$ Kronecker delta function; $\mathbf{X}_{0}=$ initial value of state vector $\mathbf{X} ; \mathbf{P}_{0}=$ variance matrix of initial value vector $\mathbf{X}_{0}$.

Through Eq. 10 and Eq. 11, a iterative process of the EKF technique can be performed in the following steps, starting with the filtered state $\hat{\mathbf{X}}_{k \mid k}$ and its error covariance matrix $\mathbf{P}_{k \mid k}$ from time $t_{k}=k \Delta t$ :

(1) Evaluating the predicted state vector and its error covariance matrix by 


$$
\begin{aligned}
& \hat{\mathbf{X}}_{k+1 \mid k}=\hat{\mathbf{X}}_{k \mid k}+\int_{t_{k}}^{t_{k+1}} \mathbf{g}[\mathbf{X}(t), \overline{\mathbf{f}}(k), t] d t \\
& \overline{\mathbf{f}}(k)=[\mathbf{f}(k)+\mathbf{f}(k+1)] / 2 \\
& \mathbf{P}_{k+1 \mid k}=\boldsymbol{\Phi}_{k} \mathbf{P}_{k \mid k} \boldsymbol{\Phi}_{k}^{\mathrm{T}}+\boldsymbol{\Gamma}_{k} \mathbf{Q}_{w, k} \boldsymbol{\Gamma}_{k}^{\mathrm{T}} / \Delta t
\end{aligned}
$$

where $\boldsymbol{\Phi}_{k}$ and $\boldsymbol{\Gamma}_{k}=$ state transition matrix and discrete noise distribution matrix of the system, respectively; for small $\Delta t$, stiffness matrix, damping matrix and input vector are assumed not to change with time, and the following approximately expression can be obtained:

$$
\begin{aligned}
& \boldsymbol{\Phi}_{k}=e^{\mathbf{A}(k) \Delta t} \approx I+\Delta t \cdot \mathbf{A}(k)=I+\Delta t \cdot[\partial \mathbf{g} / \partial \mathbf{X}]_{\mathbf{X}=\hat{\mathbf{X}}_{k k}} \\
& \boldsymbol{\Gamma}_{k}=\boldsymbol{\Phi}_{k} \cdot \mathbf{L}_{k} \cdot \Delta t
\end{aligned}
$$

(2) Calculating the Kalman gain matrix by

$$
\begin{aligned}
& \mathbf{K}_{k+1}=\mathbf{P}_{k+1 \mid k} \mathbf{H}_{k+1}^{\mathrm{T}}\left[\mathbf{H}_{k+1} \mathbf{P}_{k+|| k} \mathbf{H}_{k+1}^{\mathrm{T}}+\mathbf{R}_{k+1}\right]^{-1} \\
& \mathbf{H}_{k}=[\partial \mathbf{h} / \partial \mathbf{X}]_{\mathbf{X}=\hat{\mathbf{x}}_{k+1 \mid k}}
\end{aligned}
$$

where $\mathbf{H}_{k}=$ observation matrix of the system.

(3) Estimating the filtered state vector and its error covariance matrix by

$$
\begin{aligned}
& \hat{\mathbf{X}}_{k+1 \mid k+1}=\hat{\mathbf{X}}_{k+1 \mid k}+\mathbf{K}_{k+1}\left\{\mathbf{Z}_{k+1}-\mathbf{h}\left[\hat{\mathbf{X}}_{k+1 \mid k}, k+1\right]\right\} \\
& \mathbf{P}_{k+1 \mid k+1}=\left[\mathbf{I}-\mathbf{K}_{k+1} \mathbf{H}_{k+1}\right] \mathbf{P}_{k+1 \mid k}\left[\mathbf{I}-\mathbf{K}_{k+1} \mathbf{H}_{k+1}\right]^{\mathrm{T}}+\mathbf{K}_{k+1} \mathbf{R}_{k+1} \mathbf{K}_{k+1}^{\mathrm{T}}
\end{aligned}
$$

(4) Take the increment $k=k+1$, and return to (1) until $k=m$, where $m$ represents the discrete time $t_{m}$ for the last datum.

The above-mentioned iteration procedure, from $k=0$ to $m$, is called as a local iteration. To obtain the convergent and stable solutions, a weighted global iterative procedure with an objective function can be coupled with the local EKF procedure after the local iteration process is finished.

In the EKF-WGI procedure [13,14], the global iterations are carried out by weighting the error covariance matrix at each global iteration. To start the first global iterations, the initial values of $\hat{\boldsymbol{X}}^{(1)}(0 \mid 0)$ and $\mathbf{P}^{(1)}(0 \mid 0)$ need to be assumed, where superscript (1) represents the first global iteration. Then, using the local EKF procedure, the estimations of $\hat{\mathbf{X}}^{(1)}(m \mid m)$ and $\mathbf{P}^{(1)}(m \mid m)$ are obtained. In the second global iteration, the initial values for $\hat{\mathbf{X}}^{(2)}(0 \mid 0)$ and $\mathbf{P}^{(2)}(0 \mid 0)$ can be set to be:

$$
\hat{\mathbf{X}}^{(2)}(0 \mid 0)=\left[\begin{array}{c}
\hat{\mathbf{X}}_{1}^{(1)}(0 \mid 0) \\
\hat{\mathbf{X}}_{2}^{(1)}(0 \mid 0) \\
\hat{\mathbf{X}}_{3}^{(1)}(m \mid m) \\
\hat{\mathbf{X}}_{4}^{(1)}(m \mid m)
\end{array}\right]=\left[\begin{array}{c}
\hat{\mathbf{X}}^{(1)}(0 \mid 0) \\
\hat{\mathbf{X}}^{(1)}(0 \mid 0) \\
\hat{\mathbf{K}}^{(1)}(m \mid m) \\
\hat{\mathbf{C}}^{(1)}(m \mid m)
\end{array}\right], \mathbf{P}^{(2)}(0 \mid 0)=\left[\begin{array}{cc}
\mathbf{I} & \mathbf{0} \\
\mathbf{0} & w \mathbf{P}_{k c}^{(1)}(m \mid m)
\end{array}\right]
$$

where $w=$ weight used to accelerate the local EKF iteration; $\mathbf{P}_{k c}^{(1)}(m \mid m)=$ error covariance matrix corresponding to the parameters $\hat{\mathbf{K}}$ and $\hat{\mathbf{C}}$ at time $t_{m}$ in the last iteration. The global iterations can be repeated until convergence of the estimated state is obtained. 
To obtain the convergence of the EKF-WGI procedure, the memory fading filter technique (MFF) and Runge-Kutta method (R-K) are introduced, and an improved method, MFWKF-WGI, is proposed in this paper. In the second step, the physical parameters of the whole structure and unknown dynamic responses are estimated with limited observations and the inversed input of the first step.

Fig. 1 illustrates the flow chart of generalized hybrid inversion method.

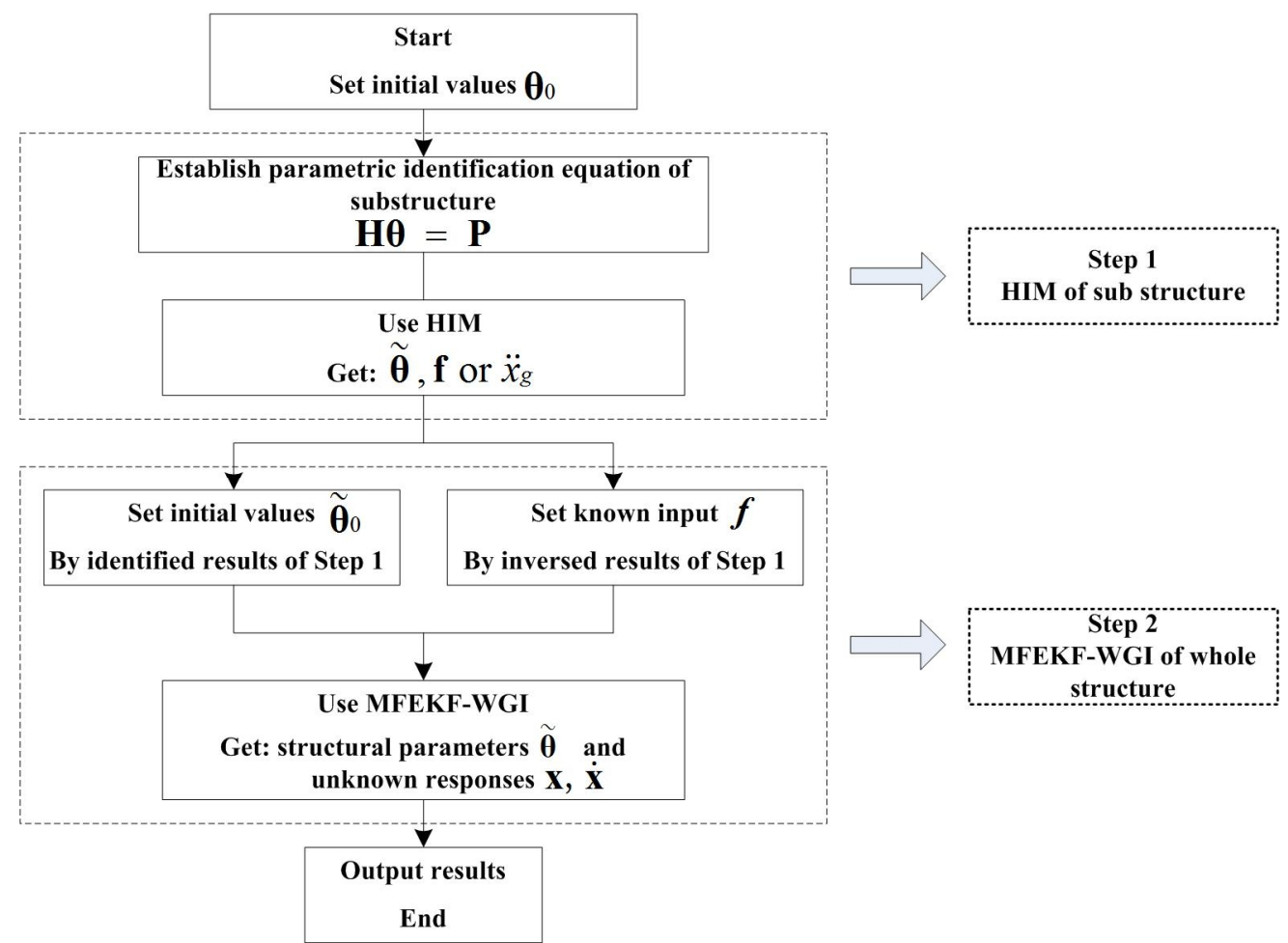

Fig. 1 Flow chart of generalized hybrid inversion method

\section{Numerical Example}

To verify the GHIM procedure, a six-story shear-type building, in Fig. 2, is considered. The actual parameter values of the 6-story building are shown in Table 1. And the damping coefficient for stiffness proportional damping, $\beta=0.020914$, is calculated using the modal analysis.

The excitation is El Centro seismic ground motion applied at the base, in Fig. 3. The theoretical structural displacement responses are simulated as the measured responses using the finite element method (FEM). Only the responses of the top three DDOFs (4th, 5th and 6th DDOF) are assumed to be measured. And after the numerical simulation the excitation is assumed to be unknown.

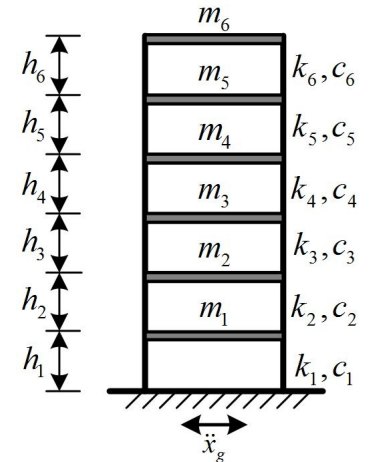

Fig. 2 Six-story shear-type structural model
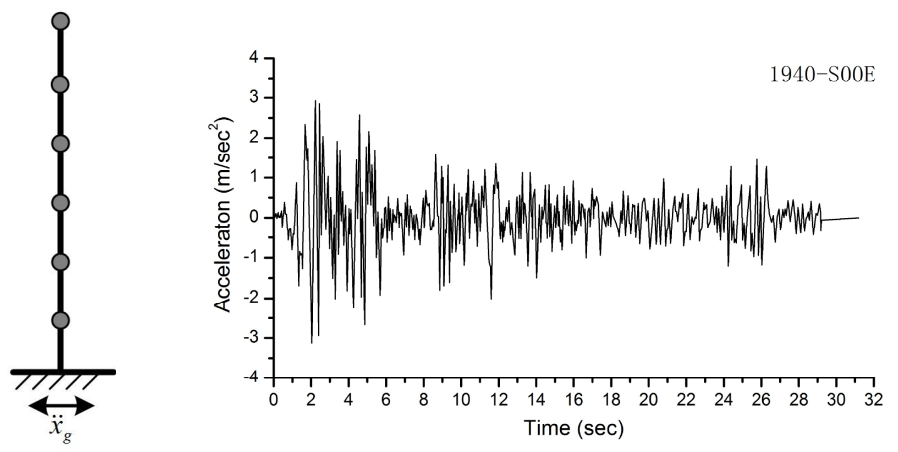

Fig. 3 Time history curve of El Centro ground motion 
Table 1 Actual parameter values of the 6-story building

\begin{tabular}{cccc}
\hline Floor & $\begin{array}{c}\text { Mass } \\
{\left[10^{5} \mathrm{~kg}\right]}\end{array}$ & $\begin{array}{c}\text { Stiffness } \\
{\left[10^{8} \mathrm{~N} / \mathrm{m}\right]}\end{array}$ & $\begin{array}{c}\text { Height } \\
{[\mathrm{m}]}\end{array}$ \\
\hline 1 & 5.0 & 1.8 & 3.0 \\
$2-3$ & 4.5 & 1.5 & 3.0 \\
$4-5$ & 4.0 & 1.2 & 3.0 \\
6 & 3.0 & 1.0 & 3.0 \\
\hline
\end{tabular}

The task is to identify the structural stiffness parameters, damping coefficient and to estimate the unmeasured responses of all DDOFs using GHIM procedure with only the responses of top three DDOFs. To investigate the effect of measurement noise on the results of state estimation, three levels of white noise are introduced into the theoretical displacement responses. The 5\% noise-contaminated responses are shown in Fig. 4. The noise level is defined as the ratio of noise's RMS (root mean square) to theoretical response's RMS, i.e. noise-to-signal ratio. The three considered noise levels are $0 \%, 1 \%$ and $5 \%$ in noise-to-signal ratio, respectively.
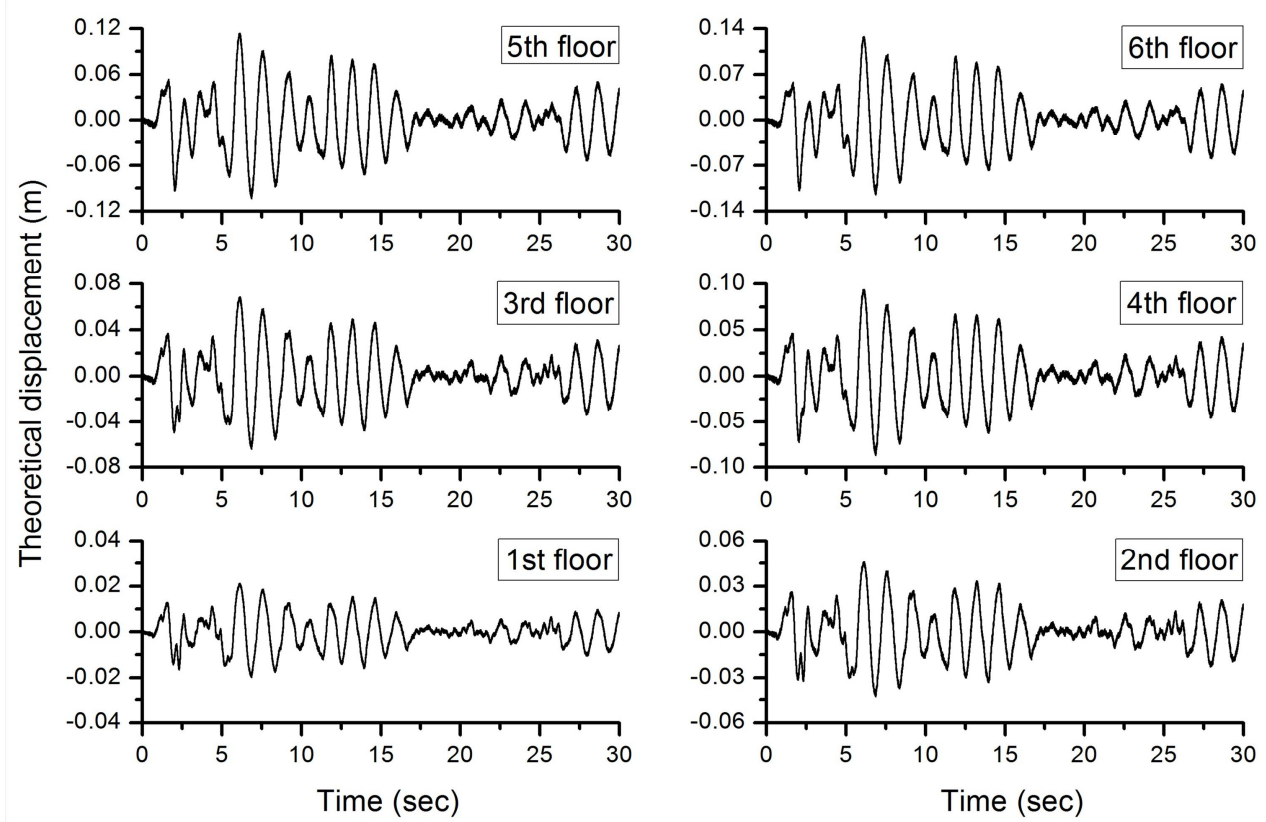

Fig. 4 Time history curves of theoretical displacement with 5\% noise-included case

First Step. The top two stories are considered to be a substructure. The stiffness parameters and damping coefficient of the substructure are identified and the ground motion is inversed using HIM. In the HIM process, the SVD-mLM method is introduced for solving the nonlinear parametric identification equation, the initial values of the structural parameters are 0.1 times of their actual values, and the rectangular window method is used for improving the results' precision with 10 windows.

The identified values of the sub-structural physical parameters are listed in Table 2. And the inversed ground motion time history is shown in Fig. 5 (with 5\% noise-contaminated case).

Table 2 The identified results of the sub-structural physical parameters (HIM)

\begin{tabular}{ccccccccc}
\hline \multirow{2}{*}{ Parameter } & \multirow{2}{*}{ Floor } & \multirow{2}{*}{ Exact } & \multicolumn{2}{c}{$0 \%$ noise } & \multicolumn{2}{c}{$1 \%$ noise } & \multicolumn{2}{c}{$5 \%$ noise } \\
\cline { 4 - 8 } & & & Results & Err. [\%] & Results & Err. [\%] & Results & Err. [\%] \\
\hline$k_{i}$ & 5 & 1.2 & 1.2000 & 0.00 & 1.1999 & -0.01 & 1.2003 & 0.02 \\
{$\left[10^{8} \mathrm{~N} / \mathrm{m}\right]$} & 6 & 1.0 & 1.0000 & 0.00 & 1.0000 & 0.00 & 1.0001 & 0.01 \\
$\beta$ & - & 0.020914 & 0.020914 & 0.00 & 0.020892 & -0.11 & 0.020837 & -0.37 \\
\hline
\end{tabular}




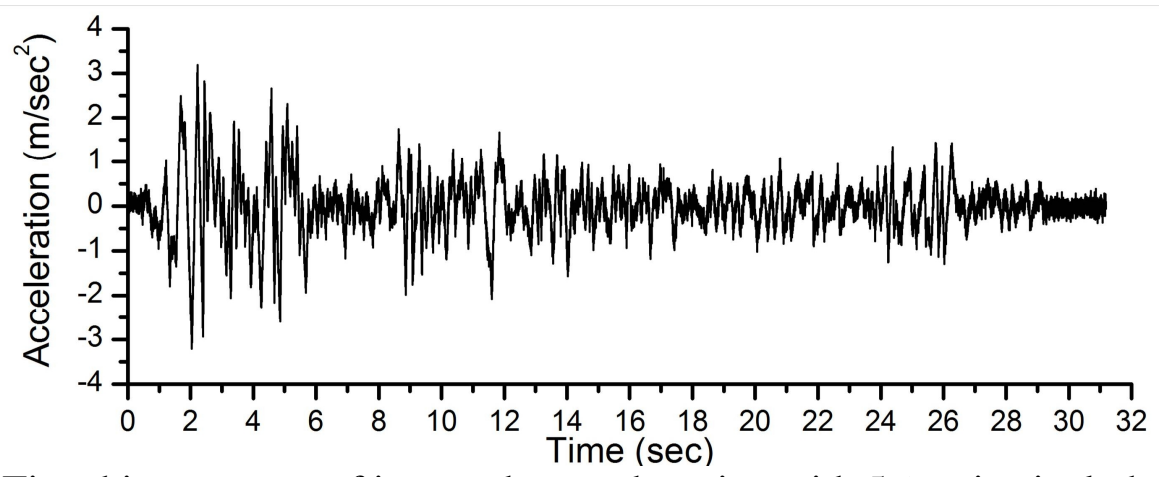

Fig. 5 Time history curve of inversed ground motion with $5 \%$ noise-included case

Second Step. The study object is the whole structure. All the structural parameters (stiffness and damping coefficient) and unknown dynamic responses are estimated using MFEKF-WGI procedure.

The state vector $(\mathbf{X})$ and the nonzero elements of the observation matrix $\left(\mathbf{H}_{6 \times 19}\right)$ are

$$
\begin{aligned}
& \mathbf{X}=\left[\begin{array}{c}
\mathbf{X}_{1} \\
\mathrm{M} \\
\mathbf{X}_{4}
\end{array}\right], \mathbf{X}_{1}=\left[\begin{array}{c}
x_{1} \\
\mathrm{M} \\
x_{6}
\end{array}\right], \mathbf{X}_{2}=\left[\begin{array}{c}
x_{1} \\
\mathrm{M} \\
\boldsymbol{x}_{6}
\end{array}\right], \mathbf{X}_{3}=\left[\begin{array}{c}
k_{1} \\
\mathrm{M} \\
k_{6}
\end{array}\right], \mathbf{X}_{4}=\beta \\
& H_{1,4}=H_{2,5}=H_{3,6}=H_{4,10}=H_{5,11}=H_{6,12}=1.0
\end{aligned}
$$

The total number of global iteration is 6 . The measured responses between $0.2-6.2 \mathrm{sec}$ are used in the first five global iterations for parameter identification, giving $m=3000$. All the measured data are used in the sixth global iteration for the estimation of the unmeasured responses and the improved identification of the parameters. The covariance matrix of system noise is $\mathbf{Q}=10^{-5} \mathbf{I}$; The covariance matrix of observation noise is $\mathbf{R}=10^{-2} \times \mathbf{I}$; The weight of global iteration is $w=1000$ or $w=100$ (with 5\% noise-contaminated case); And the weight of MFF is $S=1.002$.

In the 1 st iteration, the initial values of 5th, 6th stiffness parameters and damping coefficient are set as the identified results of Step 1, and the initial values of 1st-4th stiffness parameters are set as the 5th identified stiffness parameter of Step 1. In the 2nd-6th iteration, the initial values of parameters are set as the identified results of the previous iteration. The initial values of the state components in the velocity and displacement are zero. The initial values of the state vector and the filtered error covariance matrix are

$$
\begin{aligned}
& \hat{\mathbf{X}}^{(k+1)}(0 \mid 0)=\left[\begin{array}{c}
\hat{\mathbf{X}}_{1}^{(k)}(0 \mid c) \\
\hat{\mathbf{X}}_{2}^{(k)}(0 \mid 0) \\
\hat{\mathbf{X}}_{3}^{(k)}(L \mid c) \\
\hat{\mathbf{X}}_{4}^{(k)}(L \mid c)
\end{array}\right]=\left[\begin{array}{c}
\hat{\mathbf{x}}^{(k)}(0 \mid 0) \\
\hat{\mathbf{X}}^{(k)}(0 \mid 0) \\
\hat{\mathbf{K}}^{(k)}(L \mid L) \\
\hat{\mathbf{C}}^{(k)}(L \mid L)
\end{array}\right]=\left[\begin{array}{c}
0 \\
0 \\
\hat{\mathbf{K}}^{(k)}(L \mid L) \\
\hat{\mathbf{C}}^{(k)}(L \mid L)
\end{array}\right] \\
& \mathbf{P}^{(1)}(0 \mid 0)=\left[\begin{array}{cc}
\mathbf{I}_{12} & \mathbf{0} \\
\mathbf{0} & w \mathbf{I}_{7}
\end{array}\right] ; \quad \mathbf{P}^{(k+1)}(0 \mid 0)=\left[\begin{array}{cc}
\mathbf{I}_{12} & \mathbf{0} \\
\mathbf{0} & w \mathbf{P}_{k c}^{(k)}
\end{array}\right]
\end{aligned}
$$

The identified values of the whole structural physical parameters are listed in Table 3 . And the estimated velocity responses compared with the theoretical responses are shown in Fig. 6 (with 5\% noise- contaminated case). 
Table 3 The identified results of the whole-structural physical parameters (MFEKF-WGI)

\begin{tabular}{ccccccccc}
\hline \multirow{2}{*}{ Parameter } & \multirow{2}{*}{ Floor } & \multirow{2}{*}{ Exact } & \multicolumn{2}{c}{$0 \%$ noise } & \multicolumn{2}{c}{$1 \%$ noise } & \multicolumn{2}{c}{ 5\% noise } \\
\cline { 3 - 8 } & & & Results & Err. [\%] & Results & Err. [\%] & Results & Err. [\%] \\
\hline & 1 & 1.8 & 1.7988 & -0.07 & 1.7708 & -1.62 & 1.7228 & -4.29 \\
$k_{i}$ & 2 & 1.5 & 1.5009 & 0.06 & 1.4934 & -0.44 & 1.5351 & 2.34 \\
{$\left[10^{8} \mathrm{~N} / \mathrm{m}\right]$} & 4 & 1.5 & 1.4999 & -0.01 & 1.4966 & -0.23 & 1.4453 & -3.65 \\
& 5 & 1.2 & 1.2003 & 0.02 & 1.2248 & 2.07 & 1.2631 & 5.26 \\
& 6 & 1.2 & 1.2000 & 0.00 & 1.2009 & 0.08 & 1.2131 & 1.09 \\
$\beta$ & - & 0.020914 & 0.020914 & 0.00 & 0.020733 & -0.87 & 0.020209 & -3.37 \\
\hline
\end{tabular}
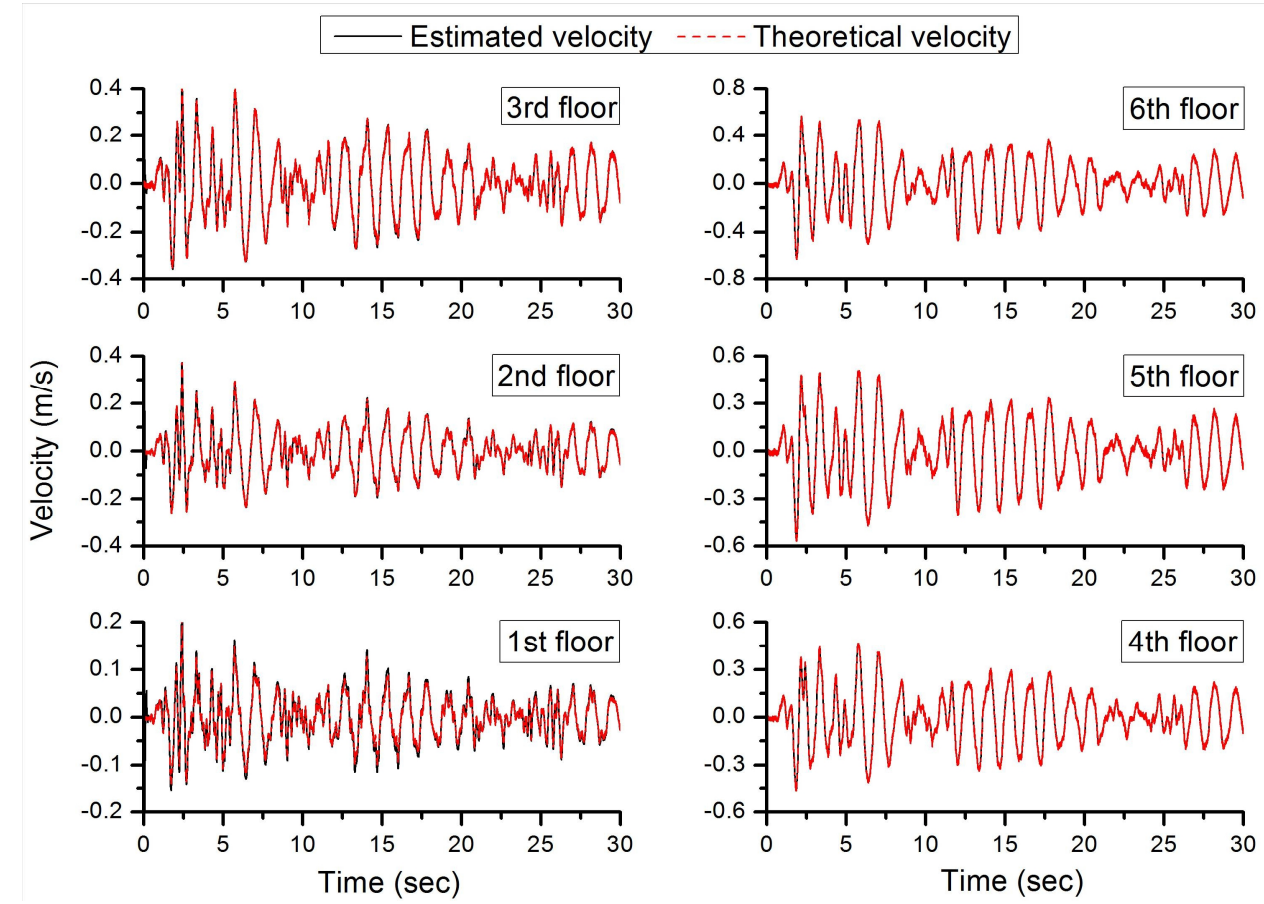

Fig. 6 Time history curves of estimated velocity vs. theoretical velocity with $5 \%$ noise-included case

In Table 2, the maximum errors of the stiffness parameters and damping coefficient of the sub-structure are $0.00 \%$ and $0.00 \%$ in the noise free case, $-0.01 \%$ and $-0.11 \%$ in the $1 \%$ noise case, and $0.02 \%$ and $-0.37 \%$ in the $5 \%$ noise case, respectively. Obviously, the HIM technique with SVD-mLM method identifies the structural physical parameters very well.

In Fig. 5, the inversed ground motion with 5 noise concluded case is illustrated. Comparing Fig. 5 with Fig. 2, the results show that the inversed ground motion agrees well with El Centro ground motion except containing noise.

In Table 3, the maximum errors of the stiffness parameters and damping coefficient of the whole-structure are $-0.07 \%$ and $0.00 \%$ in the noise free case, $2.07 \%$ and $-0.87 \%$ in the $1 \%$ noise case, and $5.26 \%$ and $-3.37 \%$ in the $5 \%$ noise case, respectively. That shows that the MFEKF-WGI procedure estimates the structural parameters very well with limited observations.

In Fig. 6, the velocity responses are estimated and compared with the theoretical responses with $5 \%$ noise contaminated case. The figure show that the estimated responses agree well with the theoretical ones. And the acceleration responses can be given using the motion equations of MDOF system, Eq. 1. So the MFEKF-WGI method estimates the unmeasured responses very well.

From the results, it is observed that the proposed GHIM procedure identified the structural physical parameters, inversed the seismic excitation at the base and estimated the unmeasured responses in time domain very well without input information and with limited observations. There are small errors in the identified parameters, and high consistent in the inversed input information and 
the estimated responses with the three noise-contaminated cases. This demonstrates the effectiveness and robustness of the GHIM method.

\section{Conclusions}

A structural physical parameter identification technique in time domain, GHIM, is proposed to identify structural parameters, inverse input information and estimate the unmeasured responses, where the measured responses are limited and the input is unknown. The method includes two steps: (1) parameter identification of sub structure and input inversion using HIM with introducing the SVD-mLM technique and the rectangular window method, and (2) estimation of whole structure's parameters and unmeasured responses using MFEKF-WGI procedure.

The method is verified using a numerical example of 6-story shear-type building. For verification purpose, three noise-included output responses (noise-free, 1\% noise-included case and 5\% noise-included case) are considered. Several important results are obtained from the numerical example. The GHIM procedure identifies the structural physical parameters, inverses the input time history and estimates the unmeasured responses very well. The errors of the identified parameters, the inversed input and the estimated responses are very small. The proposed method is accurate and robust.

In GHIM, the number of structural stories, $n$, is assumed to be larger than three. If $n \leq 3$, the structural model is very simple and efficient to identify parameters using HIM. So GHIM procedure is for large system. The results show that the SVD-mLM procedure, the rectangular window method, the MFF technique and 4th order R-K method work well and the GHIM technique is effective.

\section{Acknowledgements}

This work was financially supported by the National Science Foundation of China (51208478).

\section{References}

[1] C.S. Huang, S.L. Hung, C.M. Wen, et al.: Earthquake Engineering and Structural Dynamics, Vol. 32(2003), p.187.

[2] Y.F. Shi, C.C. Chang: Journal of Engineering Mechanics, Vol. 138(2012), p. 50.

[3] D. Rocco, M. Marco, C.P. Felice: Bulletin of Earthquake Engineering, Vol. 10(2012), p. 895.

[4] D. Wang, A. Haldar: Journal of Engineering Mechanics, Vol. 120(1994), p. 159.

[5] X.J. Wang, J. Cui: Applied Mechanics and Materials, Vol. 94-96 (2011), p. 1040.

[6] X.J. Wang, P. Wan, J. Cui: World Earthquake Engineering, Vol. 31(2015), p. 114. (in Chinese)

[7] R. Brincker, L. Zhang, and P. Andersen: Smart Materials and Structures, Vol. 10(2001), p. 441.

[8] D.M. Siringoringo and Y. Fujino: Engineering Structures, Vol. 30(2008), p. 462.

[9] S.R. Ibrahim: Journal of Spacecraft and Rockets, Vol. 14(1977), p. 696.

[10] K. Toki, T. Sato, J. Kiyono: Structural Engineering and Earthquake Engineering, Vol. 6(1989), p. 243.

[11] M. Hoshiya, E. Saito: Journal of Engineering Mechanics, ASCE, Vol. 110(1984), p. 1757.

[12] J. Li, J. Chen: Journal of Vibration and Shock, Vol. 17(1998), p. 58. (in Chinese)

[13] X.J. Wang: Study on physical parameter identification of civil engineering in time domain and inversion of ground motion (PhD Thesis, IEM, CEA, 2011). (in Chinese)

[14] D. Wang, A. Haldar: Journal of Engineering Mechanics, ASCE, Vol. 123(1997), p. 504. 